\title{
Evaluation of Dennettia tripetala Baker F. Leaf Aqueous Extract Effect on Hyperthyroidism in Albino Rats
}

\author{
$\begin{array}{lll}\text { V. C. } \text { Ejere }^{1} & \text { C. J. Iyikite } & \text { E. I. Nnamonu } \\ 2 *\end{array}$ \\ 1.Department of Zoology and Environmental Biology, University of Nigeria, Nsukka, Enugu State Nigeria \\ 2.Department of Biology, Federal College of Education, Eha-Amufu, Enugu State Nigeria
}

\begin{abstract}
This study evaluated Dennettia tripetala leaf aqueous extract effect on hyperthyroidism in albino rats (Rattus norvegicus). It was conducted at the Department of Zoology and Environmental Biology, University of Nigeria, Nsukka. It lasted for seven weeks. Seventy-two male albino rats (Rattusnovergicus) weighing 66 - $108 \mathrm{~g}$ were assigned into six groups $(\mathrm{A}-\mathrm{F})$ with twelve rats (replicated three times, four rats per replicate) in each group. Three rats from each group were randomly selected and weighed before samples of blood were collected at days 7, 14, 21 and 28. Hyperthyroidism was induced in rats of groups B - F, orally with $600 \mu \mathrm{g} / \mathrm{kg}$ Levothyroxine for 14 days. Phytochemical screening of the extract was conducted. Whereas normal control (group A) and hyperthyroid control (group B) were fed water and food only, standard control (group C) received $1.35 \mathrm{mg} / \mathrm{kg}$ of Carbimazole orally. The treatment groups were D - $100 \mathrm{mg} / \mathrm{kg}, \mathrm{E}-250 \mathrm{mg} / \mathrm{kg}$ and F $-500 \mathrm{mg} / \mathrm{kg}$. Rats'body weights were determined at days $0,7,14,21$ and 28 during treatment. The extract's effect on hyperthyroid induced rats total cholesterol (TC), triglyceride (TG), low-density lipoproteins (LDL), high-density lipoproteins (HDL), thyroid stimulating hormone (TSH), triiodothyronine (T3), tetraiodothyronine(T4) and histology of thyroid gland were evaluated. Phytochemical screening recorded some secondary metabolites. Toxicity (LD50) test recorded no lethal effect at the highest dose $(5000 \mathrm{mg} / \mathrm{kg})$ administered. The extract graded dose effect was most effective on body weight on Day 7. It also caused a significant increase $(\mathrm{P}<0.05)$ in $\mathrm{TC}, \mathrm{LDL}-\mathrm{C}, \mathrm{TG}$, and TSH but significantly decreased $(\mathrm{P}<0.05)$ HDL-C and T3 and T4 compared with hyperthyroid control. Micrographs from extract treated rats showed no histologic alteration. However, there were observed variations in body weight, LDL-C, TG, TSH and $\mathrm{T}_{4}$ the body weight at day 0 due to age differences among animals used. Aqueous Dennettiatripetala leaf extract seems to possess potential for ameliorating post induced hyperthyroidism effects in rat.
\end{abstract}

Keywords: Albino rat, Dennettia tripetala, Hyperthyroidism, Phytochemical screening, Lipid profile, Histopathology

DOI: $10.7176 / \mathrm{JNSR} / 9-8-05$

Publication date: April $30^{\text {th }} 2019$

\section{Introduction}

Hyperthyroidism encompasses a heterogeneous group of disorders characterized by elevated levels of thyroid hormones in the blood (Peter et al. 2005). It elevates body functions and formation of a toxic goiter. It elicits hyperdynamic circulation with increased cardiac output, heart rate, pulse pressure, and blood pressure, as well as decreased vascular peripheral resistance. Research has also proven that it increases metabolic rate which affects organs and system activities adversely (Golden et al. 2009).

Treatment of hyperthyroidism has largely been by chemotherapy, radiotherapy, and surgery (Bhaigyabati et al. 2012) which makes it expensive to the poor and low income earners. Due to the expensiveness of hyperthyroidism and its related diseases treatments, there is need for an alternative treatment that will be affordable to the poor and low income earners. Recently, plant-based therapy has been highly preferred to synthetic drugs which most times possess some post-treatment adverse effects and expense to many. Research has also revealed that medicinal plants are rich in secondary metabolites (Newman et al. 2003). Remarkably, some plant extracts have demonstrated hypocholesterolemic and anti-obesity potentials (Ejere \& Adegoke, 2005; Nnamonu et al. 2013; Nnamonu et al. 2018).

Dennettia tripetala belongs to the Annonaceae family. Dennettia tripetala belongs to the Annonaceae family. Dennettia tripetala is a plant commonly cultivated in the rain forest zones of Nigeria and other part of West Africa. It is some Nigeria local languages it is commonly called Nmimi (Igbo) and Ata Igbere(Yoruba) (Ihemeje et al. 2013; Iseghohi 2015). Researches have established medicinal properties of its various parts. Hypoglyceaemic, analgesic, hypolipidaemic, haematoxic and nephrotoxicity effects of Dennettia tripetala extracts have been reported (Oyemitan et al. 2008; Ikpi \& Nku, 2008; Anaga \& Asuzu 2010; Nwankpa et al. 2018). Despite the aforementioned medicinal values, there is a dearth of literature and very little is known on the possible effects of the leaf extract on treatment of hyperthyroidism from our region.In view of the foregoing, objectives ofthis study was to evaluate Dennettia tripetala leaf extract effect on hyperthyroid induced rat's total cholesterol (TC), triglyceride (TG), low density lipoproteins (LDL), high density lipoproteins (HDL), thyroid stimulating hormone (TSH), triiodothyronine (T3), tetraiodothyronine(T4) and histology of thyroid gland. 


\section{MATERIALS AND METHODS}

\subsection{Drugs and Chemicals}

Levothyroxine (Karmic Meditraders Private Limited, New Delhi, Delhi) and Carbimazole (Macleods Pharmaceuticals Ltd, India) used were purchased from reputable pharmaceutical shops at Nsukka, Enugu state, south-east Nigeria. Diagnostic kits used for determination of various lipid profile parameters were produced by Randox commercial enzyme kit while Enzyme-linked immunosorbent assays (ELISA) kits used for determination of TSH, T4, and T3 were manufactured by M.B.S./Medical Biological Service, Milano-Italy.

\subsection{Collection and preparation of Dennettia tripetala leaf aqueous extract}

Fresh leaves of $D$. tripetala were procured from a farm settlement at Ukehe, Igbo-Etiti local government area, Enugu state, southeast Nigeria. A taxonomist at the Department of Plant Science and Biotechnology, University of Nigeria, Nsukka, Nigeria identified the plant materials. The extract was prepared following the method of Oyemitan et al. 2006.

\subsection{Phytochemical Analysis}

Standard method was followed in detecting the presence of different phytochemical constituents in the extract (Case 2005).

\subsection{Lethal Dose (LD50) Determination}

Determination of the extract's lethal dose (LD50) followed the method of Lorke 1983.

\subsection{Animal management}

Seventy-two male albino rats (Rattusnovergicus) weighing $66-108 \mathrm{~g}$ were procured from the Genetics and Experimental Animal breeding Laboratory, Department of Zoology and Environmental Biology, University of Nigeria, Nsukka. They were housed in stainless wire rat cages, fed growers mash ( $18 \%$ crude protein), clean water and acclimatized for two weeks. The animals were also kept in $12 \mathrm{~h} \mathrm{light/dark} \mathrm{cycle.} \mathrm{Handling} \mathrm{of} \mathrm{animals} \mathrm{in} \mathrm{this}$ research was in accordance with that recommended by the Committee and the International Guidelines for Handling of Laboratory Animals (Derell 1996).

\subsection{Hyperthyroidism induction}

Animals in groups B - F experienced hyperthyroidism induction through oral administration of $600 \mu \mathrm{g} / \mathrm{kg}$ Levothyroxine for 14 days which was confirmed by analysing the serum thyroid hormone levels. The serum level of thyroid stimulating hormones (TSH) in hyperthyroid rats was found to have increased while the TSH decreased. The serum levels of triiodothyronine (T3) and tetraiodothyronine (T4) and TSH of the normal animal were 3.33 $\mathrm{pmol} / \mathrm{L}, 12.82 \mathrm{pmol} / \mathrm{L}$ and $2.67 \mathrm{mU} / \mathrm{L}$ respectively. Hence, rats with $5.22 \mathrm{pmol} / \mathrm{L}$ and $26.38 \mathrm{pmol} / \mathrm{L}$ or higher serum levels of T3, T4, and equivalent $0.14 \mathrm{mU} / \mathrm{L}$ serum levels of TSH or lower were regarded as hyperthyroid and subsequently used.

\subsection{Experimental design}

The rats were assigned into six groups $(\mathrm{A}-\mathrm{F})$ with twelve rats (replicated three times, four rats per replicate) in each group. Three rats from each group were randomly selected and weighed before samples of blood were collected at day 7, day 14, day 21 and day 28 .

Group A was the normal control, administered water and feed only. Group B served as hyperthyroid control (administered water, feed and $600 \mu \mathrm{g} / \mathrm{kg}$ Levothyroxine (for 14 days)) while group C (standard control) received water, feed, $600 \mu \mathrm{g} / \mathrm{kg}$ Levothyroxine and $1.35 \mathrm{mg} / \mathrm{kg}$ of Carbimazole. The treatment groups received D - 100 $\mathrm{mg} / \mathrm{kg}, \mathrm{E}-250 \mathrm{mg} / \mathrm{kg}$ and $\mathrm{F}-500 \mathrm{mg} / \mathrm{kg}$ in addition to water, feed and $600 \mu \mathrm{g} / \mathrm{kg}$ Levothyroxine. All treatments were administered orally.

\subsection{Determination of body weight and weight loss}

All the rats were weighed using a Mettler, electronic balance PC2000 at day 0 at day 0 also at each sampling day (days 7, 14, 21 and 28).

\subsection{Collection of blood sample}

About $5 \mathrm{ml}$ of the blood samples was collected from each of the anaesthetized rats using the method described by 18.

\subsection{Determination of lipid profile}

Total cholesterol, triglyceride, low-density lipoproteins (LDL) and high-density lipoproteins (HDL) were determined following Sood 2006. All lipid parameters were measured using Randox commercial enzyme kits. 

(TSH).

Determination of serum total triiodothyronine (T3), tetraiodothyronine (T4) and thyroid stimulating hormone

Serum T3, T4, and TSH were determined using Enzyme-linked immunosorbent assays (ELISAs) kits (M.B.S./Medical Biological Service, Milano-Italy).

\subsection{Histological studies}

The histological studies followed the method of Ejere et al. 2018 with minor modification. Specimens were fixed in $10 \%$ neutral buffered formalin and were processed for paraffin sections of $5 \mu \mathrm{m}$-thick stained with Haematoxylin and $\operatorname{Eosin}(\mathrm{H} \& \mathrm{E})$.

\subsection{Statistical analysis}

Data analysis was carried out with statistical package for the social sciences SPSS, IBM Statistics UK version 20.0 (one-way analysis of variance (ANOVA). The means were separated using Duncan's new multiple range tests while differences in the means were considered significant at probability values less than $5 \%(\mathrm{P}<0.05)$. Results were presented as mean \pm SEM.

\section{Results}

3.1 Qualitative and quantitative phytochemical composition of aqueous Dennettia tripetala leaf extract

Table 1(a\&b) shows results of qualitative and quantitative phytochemical compositions of the aqueous Dennettia tripetala leaf extract respectively. Qualitatively, it was observed that the aqueous $D$. tripetala leaf extract had tannin in high quantity. Similarly, it also contained alkaloids, saponins, flavonoids, reducing sugars, oil and fats, carbohydrate and cardiac glycosides in moderate quantities while acidic compound, protein, steroids, phenols, and terpenoids were detected in low quantity while resins were absent.

\section{Table 1(a \&b) Qualitative phytochemical composition of aqueous Dennettia tripetalaleaf extract}

\begin{tabular}{lc}
\hline Phytochemicals & Bioavailability \\
\hline Alkaloids & ++ \\
Tannins & ++ \\
Saponins & + \\
Acidic compounds & ++ \\
Reducing substance & + \\
Protein & ++ \\
Oil and fats & ++ \\
Carbohydrates & - \\
Resins & ++ \\
Flavonoids & ++ \\
Cardiac glycosides & + \\
Steroids & + \\
Terpenoids & + \\
Phenol & Composition (\%) \\
\hline Phytochemicals & 24 \\
Alkaloids & 58.42 \\
Tannins & 33.82 \\
Saponins & 42.02 \\
Flavonoids & 32 \\
Cardiac glycosides & 0.33 \\
Phenol &
\end{tabular}

\begin{tabular}{ll}
\hline+++ & High quantity \\
++ & Moderate quantity \\
+ & Low quantity \\
- & Absent
\end{tabular}

\subsection{Effects $D$. tripetala leaf aqueous extract on body weight $(B W)(g)$ of hyperthyroid male albino rats}

Table 2 shows the comparative effects of graded doses $(100,200$ and $500 \mathrm{mg} / \mathrm{kg})$ of aqueous leaf extract of $D$. tripetala on bodyweight of hyperthyroid male albino rats. The observed variations in the body weight at day 0 were due to age differences among animals used. No significant difference $(\mathrm{P}>0.05)$ occurred in body weight at Day 0 in all treatments except normal control that increased significantly $(\mathrm{p}<0.05)$ compared with other groups. No significant difference $(\mathrm{P}>0.05)$ was recorded on Day 7 except hyperthyroid control which decreased significantly $(\mathrm{P}<0.05)$ compared with other treatments. Similar trends occurred at Days 14 and 28 were no significant difference $(\mathrm{P}>0.05)$ occurred in all treatments. Day 21 recorded no significant difference $(\mathrm{P}>0.05)$ 
except standard control which decreased significantly $(\mathrm{P}<0.05)$ compared with other treatments. There was a timedependent significant difference $(\mathrm{P}<0.05)$ from Day 0 to Day 28 in hyperthyroid and standard control compared with normal control. The extract $(250 \mathrm{mg} / \mathrm{kg})$ caused a time-dependent significant decrease $(\mathrm{P}<0.05)$ from Day 0 to Day 7 compared with other treatments. Dose $500 \mathrm{mg} / \mathrm{kg}$ showed a time-dependent significant decrease $(\mathrm{P}<0.05)$ compared with hyperthyroid control and standard control.

Table 2 D. tripetala leaf aqueous extract on body weight (BW) (g) of hyperthyroid male albino rats

Groups

Duration

\begin{tabular}{lcrrrr} 
& \multicolumn{1}{c}{ Day 0 } & \multicolumn{1}{c}{ Day 7 } & \multicolumn{1}{c}{ Day 14 } & \multicolumn{1}{c}{ Day 21 } & \multicolumn{1}{c}{ Day 28 } \\
\cline { 2 - 6 } Normal Control & $103.92 \pm 4.58^{\mathrm{b} 1}$ & $104.42 \pm 10.00^{\mathrm{ab} 1}$ & $111.00 \pm 7.82^{\mathrm{a} 1}$ & $119.88 \pm 10.31^{\mathrm{ab} 1}$ & $130.61 \pm 19.39^{\mathrm{a} 1}$ \\
Hyperthyroid & $73.30 \pm 3.51^{\mathrm{a} 1}$ & $87.14 \pm 1.92^{\mathrm{a} 2}$ & $107.03 \pm 2.68^{\mathrm{a} 3}$ & $124.76 \pm 7.49^{\mathrm{ab} 4}$ & $149.75 \pm 1.52^{\mathrm{a} 5}$ \\
Control & $79.22 \pm 2.80^{\mathrm{a} 1}$ & $98.02 \pm 8.40^{\mathrm{ab} 2}$ & $113.30 \pm 5.03^{\mathrm{a} 3}$ & $113.67 \pm 0.90^{\mathrm{a} 3}$ & $152.68 \pm 0.55^{\mathrm{a} 4}$ \\
Standard Control & $74.75 \pm 2.40^{\mathrm{a} 1}$ & $110.21 \pm 5.20^{\mathrm{b} 2}$ & $125.39 \pm 8.61^{\mathrm{a} 23}$ & $141.75 \pm 6.15^{\mathrm{b} 34}$ & $155.48 \pm 5.81^{\mathrm{a} 4}$ \\
$100 \mathrm{mg} / \mathrm{kg}$ & $79.76 \pm 1.60^{\mathrm{a} 1}$ & $97.76 \pm 1.20^{\mathrm{ab} 1}$ & $115.41 \pm 8.33^{\mathrm{a} 12}$ & $139.04 \pm 12.55^{\mathrm{ab} 23}$ & $154.95 \pm 19.73^{\mathrm{a} 3}$ \\
$250 \mathrm{mg} / \mathrm{kg}$ & $78.43 \pm 6.05^{\mathrm{a} 1}$ & $115.31 \pm 2.61^{\mathrm{b} 12}$ & $131.19 \pm 19.93^{\mathrm{a} 2}$ & $137.03 \pm 6.48^{\mathrm{ab} 2}$ & $143.91 \pm 18.82^{\mathrm{a} 2}$ \\
$500 \mathrm{mg} / \mathrm{kg}$ & & &
\end{tabular}

Values represent mean \pm SEM of three observations. Values represent mean \pm SEM of three observations. Values with different alphabet superscript in a column were significantly different $(\mathrm{P}<0.05)$. Values with different figures superscript across a row were significantly different $(\mathrm{P}<0.05)$.

3.3 Effects D. tripetala leaf aqueous extract on the T3 levels (pmol/L) of hyperthyroid male albino rats The comparative effects of graded doses $(100,250$ and $500 \mathrm{mg} / \mathrm{kg})$ of aqueous leaf extract of $D$. tripetala on T3 levels of hyperthyroid male albino rats were shown in Table 3. The extract's graded doses (100, 250 and 500 $\mathrm{mg} / \mathrm{kg}$ ) caused a significant decrease $(\mathrm{P}<0.05)$ in $\mathrm{T} 3$ levels compared with hyperthyroid control (Day 7 to 28).

A time-independent significant decrease $(\mathrm{P}<0.05)$ occurred in treatments $(250$ and $500 \mathrm{mg} / \mathrm{kg})$ from Day 0 to Day 28 compared with hyperthyroid controls.

Table 3 D. tripetala leaf aqueous extract on the $T_{3}$ levels (pmol/L) of hyperthyroid male albino rats

\begin{tabular}{lcrrrr}
\hline \multirow{2}{*}{\multicolumn{5}{c}{ Groups }} & \multicolumn{5}{c}{ Duration } \\
\cline { 2 - 6 } & \multicolumn{1}{c}{ Day 0 } & \multicolumn{1}{c}{ Day 7 } & \multicolumn{1}{c}{ Day 14 } & \multicolumn{1}{c}{ Day 21 } & Day 28 \\
\hline Normal Control & $3.33 \pm 0.23^{\mathrm{a} 1}$ & $3.25 \pm 0.11^{\mathrm{a} 1}$ & $3.20 \pm 0.34^{\mathrm{a} 1}$ & $3.32 \pm 0.22^{\mathrm{a} 1}$ & $3.11 \pm 0.11^{\mathrm{a} 1}$ \\
Hyperthyroid & $6.47 \pm 0.90^{\mathrm{ab} 1}$ & $7.97 \pm 1.46^{\mathrm{b} 12}$ & $10.18 \pm 0.56^{\mathrm{c} 2}$ & $8.70 \pm 0.53^{\mathrm{b} 12}$ & $8.42 \pm 0.41^{\mathrm{c} 12}$ \\
Control & $6.33 \pm 1.15^{\mathrm{ab} 2}$ & $4.35 \pm 0.39^{\mathrm{a} 1}$ & $4.39 \pm 0.47^{\mathrm{a} 1}$ & $3.45 \pm 0.22^{\mathrm{a} 1}$ & $2.77 \pm 0.21^{\mathrm{a} 1}$ \\
Standard Control & $6.33 \pm 1.11^{\mathrm{ab} 2}$ & $6.61 \pm 0.38^{\mathrm{b} 2}$ & $6.89 \pm 1.21^{\mathrm{b} 2}$ & $3.78 \pm 0.29^{\mathrm{a} 1}$ & $5.11 \pm 0.11^{\mathrm{b} 12}$ \\
$100 \mathrm{mg} / \mathrm{kg}$ & $5.22 \pm 0.07^{\mathrm{ab} 4}$ & $4.41 \pm 0.35^{\mathrm{a} 23}$ & $4.94 \pm 0.14^{\mathrm{a} 34}$ & $3.74 \pm 0.29^{\mathrm{a} 12}$ & $3.20 \pm 0.10^{\mathrm{a} 1}$ \\
$250 \mathrm{mg} / \mathrm{kg}$ & $6.16 \pm 1.19^{\mathrm{ab} 2}$ & $3.86 \pm 0.32^{\mathrm{a} 1}$ & $4.49 \pm 0.35^{\mathrm{a} 12}$ & $3.09 \pm 0.12^{\mathrm{a} 1}$ & $3.14 \pm 0.10^{\mathrm{a} 1}$ \\
$500 \mathrm{mg} / \mathrm{kg}$ & & &
\end{tabular}

Values represent mean \pm SEM of three observations. Values represent mean \pm SEM of three observations. Values with different alphabet superscript in a column were significantly different $(\mathrm{P}<0.05)$. Values with different figures superscript across a row were significantly different $(\mathrm{P}<0.05)$.

3.4 Effects of D. tripetala leaf aqueous extract on the T4 levels (pmol/L) of hyperthyroid male albino rats Table 4 showed the comparative effects of graded doses $(100,250$ and $500 \mathrm{mg} / \mathrm{kg})$ of aqueous leaf extract of $D$. tripetala on T4 levels of hyperthyroid male albino rats. The observed variations in T4 levels at day 0 were due to age differences among animals used. There was a dose-dependent significant decrease $(\mathrm{P}<0.05)$ in T4 levels caused by the activities of the extract's graded doses $(100,250$ and $500 \mathrm{mg} / \mathrm{kg})$ at days 14,21 and 28 compared with hyperthyroid control.

There was an observed time-dependent significant decrease caused by the extract $(100,250$ and $500 \mathrm{mg} / \mathrm{kg})$ from 7 to 14 and day 21 to 28 compared with hyperthyroid control. 
Table 4 D. tripetala leaf aqueous extract on the $\mathrm{T}_{4}$ levels $(\mathrm{pmol} / \mathrm{L})$ of hyperthyroid male albino rats

\begin{tabular}{|c|c|c|c|c|c|}
\hline \multirow{2}{*}{ Groups } & \multicolumn{5}{|c|}{ Duration } \\
\hline & Day 0 & Day 7 & Day 14 & Day 21 & Day 28 \\
\hline Normal Control & $12.82 \pm 0.82^{\mathrm{a} 1}$ & $13.39 \pm 0.80^{\mathrm{a} 1}$ & $14.19 \pm 0.62^{\mathrm{a} 1}$ & $13.36 \pm 0.37^{\mathrm{a} 1}$ & $13.01 \pm 0.63^{\mathrm{a} 1}$ \\
\hline $\begin{array}{l}\text { Hyperthyroid } \\
\text { Control }\end{array}$ & $29.55 \pm 1.86^{\mathrm{bc} 12}$ & $22.70 \pm 3.26^{\mathrm{b} 1}$ & $33.22 \pm 0.47^{\mathrm{d} 2}$ & $29.26 \pm 0.91^{\mathrm{c} 12}$ & $27.27 \pm 3.52^{\mathrm{c} 12}$ \\
\hline Standard Control & $31.91 \pm 1.65^{\mathrm{c} 3}$ & $21.08 \pm 1.46^{\mathrm{b} 2}$ & $19.54 \pm 1.13^{\mathrm{b} 2}$ & $17.06 \pm 1.43^{\mathrm{b} 12}$ & $14.81 \pm 0.54^{\mathrm{ab} 1}$ \\
\hline $100 \mathrm{mg} / \mathrm{kg}$ & $26.38 \pm 1.29^{\mathrm{b} 3}$ & $21.68 \pm 2.11^{\mathrm{b} 123}$ & $23.77 \pm 1.76^{\mathrm{c} 23}$ & $19.16 \pm 1.07^{\mathrm{b} 12}$ & $18.48 \pm 0.48^{\mathrm{b} 1}$ \\
\hline $250 \mathrm{mg} / \mathrm{kg}$ & $28.71 \pm 2.15^{\text {bc } 2}$ & $21.57 \pm 3.41^{\mathrm{b} 1}$ & $21.36 \pm 0.38^{\mathrm{bc} 1}$ & $18.07 \pm 0.59^{\mathrm{b} 1}$ & $18.92 \pm 0.75^{\mathrm{b} 1}$ \\
\hline $500 \mathrm{mg} / \mathrm{kg}$ & $28.83 \pm 1.58^{\mathrm{bc} 3}$ & $23.06 \pm 2.66^{\mathrm{b} 2}$ & $20.51 \pm 0.52^{\mathrm{b} 2}$ & $18.20 \pm 1.10^{\mathrm{b} 12}$ & $15.39 \pm 0.26^{\mathrm{ab} 1}$ \\
\hline
\end{tabular}

Values represent mean \pm SEM of three observations. Values represent mean \pm SEM of three observations. Values with different alphabet superscript in a column were significantly different $(\mathrm{P}<0.05)$. Values with different figures superscript across a row were significantly different $(\mathrm{P}<0.05)$.

3.5 Effects of D. tripetala leaf aqueous extract on the thyroid stimulating hormone (TSH) (mU/L) level of hyperthyroid male albino rats

The comparative effects of the increasing doses $(100,250$ and $500 \mathrm{mg} / \mathrm{kg})$ of the aqueous leaf extract of $D$. tripetala on TSH levels of hyperthyroid male albino rats are shown in Table 5. The observed variations in TSH levels at day 0 was due to age differences among animals used. The extract treatment recorded significant increases at 100 $\mathrm{mg} / \mathrm{kg}$ (days 7 to 28 ) and 250 and $500 \mathrm{mg} / \mathrm{kg}$ (days 14 to 28) compared with hyperthyroid control.

No time-dependent significant difference $(\mathrm{P}>0.05)$ was observed in all graded doses from Day 0 to Day 7 when compared with control groups. However, the $500 \mathrm{mg} / \mathrm{kg}$ treatment group showed a time-dependent significant increase $(\mathrm{P}<0.05)$ from Days 7 and 14 as comparison with controls.

Table $5 \mathrm{D}$. tripetala leaf aqueous extract on the thyroxin stimulating hormone (TSH) (mU/L) level of hyperthyroid male albino rats

\begin{tabular}{lccccc}
\multicolumn{1}{c}{ Groups } & \multicolumn{5}{c}{ Duration } \\
\cline { 2 - 6 } & \multicolumn{1}{c}{ Day 0 } & \multicolumn{1}{c}{ Day 7} & \multicolumn{1}{c}{ Day 14 } & Day 21 & Day 28 \\
\hline Normal Control & $2.67 \pm 0.30^{\mathrm{b} 1}$ & $2.71 \pm 0.45^{\mathrm{b} 1}$ & $2.63 \pm 0.42^{\mathrm{b} 1}$ & $3.12 \pm 0.07^{\mathrm{b} 1}$ & $2.82 \pm 0.55^{\mathrm{b} 1}$ \\
Hyperthyroid & $0.10 \pm 0.02^{\mathrm{a} 1}$ & $1.08 \pm 0.08^{\mathrm{a} 2}$ & $0.34 \pm 0.22^{\mathrm{a} 1}$ & $0.09 \pm 0.02^{\mathrm{a} 1}$ & $0.09 \pm 0.01^{\mathrm{a} 1}$ \\
Control & $0.13 \pm 0.03^{\mathrm{a} 1}$ & $2.81 \pm 0.31^{\mathrm{b} 2}$ & $3.42 \pm 0.35^{\mathrm{bc} 2}$ & $3.17 \pm 0.23^{\mathrm{b} 2}$ & $3.20 \pm 0.44^{\mathrm{b} 2}$ \\
Standard Control & $0.14 \pm 0.07^{\mathrm{a} 1}$ & $2.58 \pm 0.33^{\mathrm{b} 2}$ & $2.84 \pm 0.32^{\mathrm{bc} 23}$ & $3.51 \pm 0.44^{\mathrm{b} 23}$ & $3.69 \pm 0.30^{\mathrm{b} 3}$ \\
$100 \mathrm{mg} / \mathrm{kg}$ & $0.12 \pm 0.05^{\mathrm{a} 1}$ & $2.19 \pm 0.57^{\mathrm{ab} 2}$ & $3.19 \pm 0.07^{\mathrm{bc} 23}$ & $3.67 \pm 0.34^{\mathrm{b} 3}$ & $3.70 \pm 0.26^{\mathrm{b} 3}$ \\
$250 \mathrm{mg} / \mathrm{kg}$ & $0.12 \pm 0.03^{\mathrm{a} 1}$ & $2.15 \pm 0.57^{\mathrm{ab} 2}$ & $3.76 \pm 0.34^{\mathrm{c} 3}$ & $3.63 \pm 0.35^{\mathrm{b} 3}$ & $3.29 \pm 0.29^{\mathrm{b} 3}$ \\
$500 \mathrm{mg} / \mathrm{kg}$ & &
\end{tabular}

Values represent mean \pm SEM of three observations. Values with different alphabet superscript in a column were significantly different $(\mathrm{P}<0.05)$. Values with different figures superscript across a row were significantly different $(\mathrm{P}<0.05)$.

\subsection{Effects of D. tripetala leaf aqueous extract on the total cholesterol level ( $\mathrm{mg} / \mathrm{dL}$ ) of hyperthyroid male albino rats}

Table 6 shows the comparative effects of increasing doses $(100,250$ and $500 \mathrm{mg} / \mathrm{kg})$ of $D$. tripetala aqueous leaf extract on total cholesterol level of hyperthyroid male albino rats. No significant difference $(\mathrm{P}>0.05)$ was observed at Days 0 and 7 compared with controls. However, days 14 to 28 recorded significant increase $(\mathrm{P}<0.05)$ in total cholesterol in extract graded doses $(100,250$ and $500 \mathrm{mg} / \mathrm{kg})$ compared with the hyperthyroid control.

No time-dependent significant difference $(\mathrm{P}>0.05)$ was observed at all graded doses $(100,250$ and $500 \mathrm{mg} / \mathrm{kg})$ from days 0 to 28 compared with controls. 
Table 6 D. tripetala leaf aqueous extract on the total cholesterol level (mg/dL) of hyperthyroid male albino rats

\begin{tabular}{|c|c|c|c|c|c|}
\hline \multirow{2}{*}{ Groups } & \multicolumn{5}{|c|}{ Duration } \\
\hline & Day 0 & Day 7 & Day 14 & Day 21 & Day 28 \\
\hline Normal Control & $46.47 \pm 3.27^{\mathrm{a} 1}$ & $58.13 \pm 1.60^{\mathrm{a} 12}$ & $58.57 \pm 6.56^{\mathrm{b} 12}$ & $70.73 \pm 7.78^{\mathrm{ab} 23}$ & $79.17 \pm 2.96^{\mathrm{b} 3}$ \\
\hline $\begin{array}{l}\text { Hyperthyroid } \\
\text { Control }\end{array}$ & $46.20 \pm 2.08^{\mathrm{a} 1}$ & $78.20 \pm 2.17^{\mathrm{b} 2}$ & $42.97 \pm 0.75^{\mathrm{a} 1}$ & $46.20 \pm 1.68^{\mathrm{a} 1}$ & $47.50 \pm 5.85^{\mathrm{a} 1}$ \\
\hline Standard Control & $48.17 \pm 2.92^{\mathrm{a} 1}$ & $68.07 \pm 4.62^{\mathrm{ab} 12}$ & $81.60 \pm 5.54^{\mathrm{c} 2}$ & $109.43 \pm 10.96^{\mathrm{c} 3}$ & $112.70 \pm 14.84^{\mathrm{c} 3}$ \\
\hline $100 \mathrm{mg} / \mathrm{kg}$ & $48.83 \pm 2.67^{\mathrm{a} 1}$ & $73.80 \pm 3.73^{\mathrm{b} 2}$ & $73.00 \pm 3.86^{\mathrm{bc} 2}$ & $56.50 \pm 4.10^{\mathrm{a} 12}$ & $72.20 \pm 8.95^{\mathrm{ab} 2}$ \\
\hline $250 \mathrm{mg} / \mathrm{kg}$ & $50.23 \pm 2.03^{\mathrm{a} 1}$ & $69.63 \pm 5.74^{\mathrm{ab} 12}$ & $77.47 \pm 5.34^{\mathrm{c} 12}$ & $99.50 \pm 14.59^{\mathrm{bc} 2}$ & $97.37 \pm 13.71^{\mathrm{bc} 2}$ \\
\hline $500 \mathrm{mg} / \mathrm{kg}$ & $53.43 \pm 8.14^{\mathrm{a} 1}$ & $72.60 \pm 6.49^{\mathrm{ab} 12}$ & $85.97 \pm 5.12^{\mathrm{c} 2}$ & $119.23 \pm 11.40^{\mathrm{c} 3}$ & $98.40 \pm 10.64^{\mathrm{bc} 23}$ \\
\hline
\end{tabular}

Values represent mean \pm SEM of three observations. Values with different alphabet superscript in a column were significantly different $(\mathrm{P}<0.05)$. Values with different figures superscript across a row were significantly different $(\mathrm{P}<0.05)$.

3.7 Effects of D. tripetala leaf aqueous extract on the High-Density Lipoprotein Cholesterol (HDL-C) levels ( $\mathrm{mg} / \mathrm{dL}$ ) of hyperthyroid male albino rats

The comparative effects of graded doses $(100,250$ and $500 \mathrm{mg} / \mathrm{kg})$ of aqueous leaf extract of $D$. tripetala on serum HDL-C levels of hyperthyroid male albino rats are shown in Table 7. No significant difference $(\mathrm{P}>0.05)$ was observed at Day 0. There was an observed significant decrease $(\mathrm{P}<0.05)$ in HDL-C caused by the activities of the extract's graded doses $(100,250$ and $500 \mathrm{mg} / \mathrm{kg})$ as a comparison with hyperthyroid control.

There was no time-dependent significant difference $(\mathrm{P}>0.05)$ at all treatments compared with controls.

Table 7 D. tripetala leaf aqueous extract on the high density lipoproteins (HDL-C) levels (mg/dL) of hyperthyroid male albino rats

\begin{tabular}{|c|c|c|c|c|c|}
\hline \multirow{2}{*}{ Groups } & \multicolumn{5}{|c|}{ Duration } \\
\hline & Day 0 & Day 7 & Day 14 & Day 21 & Day 28 \\
\hline Normal Control & $43.83 \pm 2.94^{\mathrm{a} 2}$ & $26.07 \pm 1.09^{\mathrm{a} 1}$ & $27.10 \pm 2.06^{\mathrm{ab} 1}$ & $31.10 \pm 1.59^{\mathrm{b} 1}$ & $29.60 \pm 0.92^{\mathrm{a} 1}$ \\
\hline $\begin{array}{l}\text { Hyperthyroid } \\
\text { Control }\end{array}$ & $43.93 \pm 6.08^{\mathrm{a} 1}$ & $42.53 \pm 2.20^{\mathrm{c} 1}$ & $49.63 \pm 2.74^{\mathrm{c} 1}$ & $39.70 \pm 4.74^{\mathrm{c} 1}$ & $51.23 \pm 1.99^{\mathrm{b} 1}$ \\
\hline Standard Control & $45.33 \pm 4.10^{\mathrm{a} 4}$ & $32.33 \pm 1.02^{\mathrm{b} 23}$ & $28.10 \pm 1.26^{\mathrm{ab} 12}$ & $23.10 \pm 1.12^{\mathrm{ab} 1}$ & $35.93 \pm 2.25^{\mathrm{a} 3}$ \\
\hline $100 \mathrm{mg} / \mathrm{kg}$ & $45.87 \pm 6.67^{\mathrm{a} 2}$ & $33.60 \pm 1.81^{\mathrm{b} 1}$ & $31.43 \pm 0.58^{\mathrm{b} 1}$ & $27.50 \pm 1.36^{\mathrm{ab} 1}$ & $35.77 \pm 1.42^{\mathrm{a} 12}$ \\
\hline $250 \mathrm{mg} / \mathrm{kg}$ & $37.50 \pm 4.45^{\mathrm{a} 2}$ & $33.33 \pm 0.35^{\mathrm{b} 12}$ & $28.17 \pm 1.45^{\mathrm{ab} 1}$ & $27.83 \pm 3.01^{\mathrm{ab} 1}$ & $36.43 \pm 1.39^{\mathrm{a} 12}$ \\
\hline $500 \mathrm{mg} / \mathrm{kg}$ & $37.87 \pm 4.54^{\mathrm{a} 3}$ & $30.80 \pm 0.43^{\mathrm{b} 23}$ & $24.07 \pm 1.27^{\mathrm{a} 12}$ & $19.90 \pm 1.18^{\mathrm{a} 1}$ & $32.63 \pm 4.43^{\mathrm{a} 23}$ \\
\hline
\end{tabular}

Values represent mean \pm SEM of three observations. Values with different alphabet superscript in a column were significantly different $(\mathrm{P}<0.05)$. Values with different figures superscript across a row were significantly different $(\mathrm{P}<0.05)$.

3.8 Effects of D. tripetala leaf aqueous extract on the Low-Density Lipoprotein Cholesterol (LDL-C) levels (mg/dL) of hyperthyroid male albino rats

Table 8 shows the comparative effects of increasing doses $(100,250$ and $500 \mathrm{mg} / \mathrm{kg})$ of aqueous leaf extract of $D$. tripetala on low-density lipoprotein cholesterol levels of hyperthyroid male albino rats. The observed variations in LDL-C levels at day 0 were due to age differences among the animals used. No significant difference $(\mathrm{P}>0.05)$ in LDL-C levels at all treatments compared with the controls. 
Table 8 D. tripetala leaf aqueous extract on the low density lipoproteins (LDL-C) levels (mg/dL) of hyperthyroid male albino rats

\begin{tabular}{lccrrr}
\multirow{2}{*}{ Groups } & \multicolumn{5}{c}{ Duration } \\
\cline { 2 - 6 } & \multicolumn{1}{c}{ Day 0 } & \multicolumn{1}{c}{ Day 7} & \multicolumn{1}{c}{ Day 14 } & \multicolumn{1}{c}{ Day 21 } & Day 28 \\
\hline Normal Control & $31.33 \pm 8.35^{\mathrm{b} 2}$ & $19.00 \pm 0.58^{\mathrm{ab} 12}$ & $18.33 \pm 0.67^{\mathrm{a} 12}$ & $17.67 \pm 0.33^{\mathrm{a} 1}$ & $18.67 \pm 2.03^{\mathrm{a} 12}$ \\
Hyperthyroid & $18.33 \pm 1.20^{\mathrm{a} 1}$ & $22.00 \pm 1.15^{\mathrm{abc} 1}$ & $27.33 \pm 2.91^{\mathrm{ab} 12}$ & $25.67 \pm 3.28^{\mathrm{a} 12}$ & $34.33 \pm 6.81^{\mathrm{ab} 2}$ \\
Control & & & & \\
Standard Control & $18.33 \pm 1.20^{\mathrm{a} 1}$ & $23.00 \pm 0.58^{\mathrm{bc} 1}$ & $48.00 \pm 2.00^{\mathrm{d} 2}$ & $64.33 \pm 6.39^{\mathrm{cd} 2}$ & $67.00 \pm 12.70^{\mathrm{de} 2}$ \\
$100 \mathrm{mg} / \mathrm{kg}$ & $17.67 \pm 0.33^{\mathrm{a} 1}$ & $19.67 \pm 1.20^{\mathrm{ab} 1}$ & $33.67 \pm 3.18^{\mathrm{bc} 12}$ & $45.33 \pm 7.31^{\mathrm{b} 2}$ & $45.67 \pm 7.6^{\mathrm{bc} 2}$ \\
$250 \mathrm{mg} / \mathrm{kg}$ & $16.67 \pm 0.88^{\mathrm{a} 1}$ & $25.33 \pm 0.88^{\mathrm{c} 2}$ & $40.00 \pm 1.15^{\mathrm{cd} 3}$ & $54.00 \pm 5.20^{\mathrm{bc} 4}$ & $56.67 \pm 1.76^{\mathrm{cd} 4}$ \\
$500 \mathrm{mg} / \mathrm{kg}$ & $16.33 \pm 1.20^{\mathrm{a} 1}$ & $18.33 \pm 2.33^{\mathrm{a} 1}$ & $46.67 \pm 5.55^{\mathrm{d} 2}$ & $75.67 \pm 4.18^{\mathrm{d} 3}$ & $82.00 \pm 3.21^{\mathrm{e} 3}$ \\
\hline
\end{tabular}

Values represent mean \pm SEM of three observations. Values with different alphabet superscript in a column were significantly different $(\mathrm{P}<0.05)$. Values with different figures superscript across a row were significantly different $(\mathrm{P}<0.05)$.

\subsection{Effects of D. tripetala leaf aqueous extract on the triglycerides (TG) levels $(\mathrm{mg} / \mathrm{dL})$ of hyperthyroid male albino rats}

The comparative effects of graded doses $(100,250$ and $500 \mathrm{mg} / \mathrm{kg})$ of aqueous leaf extract of $D$. tripetala on serum triglycerides (TG) levels of hyperthyroid male albino rats are shown in Table 9. The observed variations in TG levels at day 0 were due to age differences among the animals used. The $100 \mathrm{mg} / \mathrm{kg}$ extract treatment caused a significant decrease $(\mathrm{P}<0.05)$ and increase $(\mathrm{P}<0.05)$ in $\mathrm{TG}$ at days 7 and 28 respectively compared with hyperthyroid control. Whereas 250 and $500 \mathrm{mg} / \mathrm{kg}$ treatments caused no significant difference $(\mathrm{P}>0.05)$ days 7 to 14 , they significantly increased $(\mathrm{P}<0.05)$ TG levels days 21 to 28 compared with hyperthyroid control.

Secondly, it was observed that treatment with $100 \mathrm{mg} / \mathrm{kg}$ showed no time-dependent significant difference ( $\mathrm{P}>0.05)$ compared with all controls. On the other hand, the treatment doses of 250 and $500 \mathrm{mg} / \mathrm{kg}$ showed a timedependent significant increase $(\mathrm{P}<0.05)$ in Days $0,7,14$ and 21 respectively when compared with controls.

Table 9 D. tripetala leaf aqueous extract on the triglycerides (TG) levels (mg/dL) of hyperthyroid male albino rats

\begin{tabular}{lccccc}
\hline \multirow{2}{*}{ Groups } & \multicolumn{5}{c}{ Duration } \\
\cline { 2 - 6 } & \multicolumn{1}{c}{ Day 0 } & Day 7 & Day 14 & Day 21 & Day 28 \\
\hline $\begin{array}{l}\text { Normal } \\
\text { Control }\end{array}$ & $49.07 \pm 4.68^{\mathrm{ab} 1}$ & $63.17 \pm 4.44^{\mathrm{ab} 12}$ & $66.53 \pm 3.28^{\mathrm{ab} 12}$ & $72.40 \pm 10.19^{\mathrm{ab} 2}$ & $60.47 \pm 3.77^{\mathrm{bc} 12}$ \\
Hyperthyroid & & & & \\
Control & $40.73 \pm 1.79^{\mathrm{a} 1}$ & $70.73 \pm 6.23^{\mathrm{b} 2}$ & $38.70 \pm 4.39^{\mathrm{a} 1}$ & $31.37 \pm 1.42^{\mathrm{a} 1}$ & $33.23 \pm 1.90^{\mathrm{a} 1}$ \\
Standard & & & & \\
Control & $44.27 \pm 5.00^{\mathrm{ab} 1}$ & $65.90 \pm 7.21^{\mathrm{b} 1}$ & $79.00 \pm 5.47^{\mathrm{b} 12}$ & $64.63 \pm 26.34^{\mathrm{ab} 1}$ & $111.20 \pm 10.35^{\mathrm{d} 2}$ \\
$100 \mathrm{mg} / \mathrm{kg}$ & $52.27 \pm 3.04^{\mathrm{b} 1}$ & $46.63 \pm 0.67^{\mathrm{a} 1}$ & $49.97 \pm 22.90^{\mathrm{ab} 1}$ & $53.87 \pm 3.14^{\mathrm{a} 1}$ & $54.50 \pm 4.43^{\mathrm{b} 1}$ \\
$250 \mathrm{mg} / \mathrm{kg}$ & $46.47 \pm 1.56^{\mathrm{ab} 1}$ & $62.37 \pm 11.71^{\mathrm{ab} 12}$ & $68.23 \pm 6.05^{\mathrm{ab} 12}$ & $98.57 \pm 6.47^{\mathrm{b} 3}$ & $77.30 \pm 11.02^{\mathrm{c} 23}$ \\
$500 \mathrm{mg} / \mathrm{kg}$ & $45.23 \pm 1.07^{\mathrm{ab} 1}$ & $72.47 \pm 3.26^{\mathrm{b} 2}$ & $69.13 \pm 8.01^{\mathrm{ab} 2}$ & $105.77 \pm 9.12^{\mathrm{b} 3}$ & $106.53 \pm 4.42^{\mathrm{d} 3}$ \\
\hline
\end{tabular}

Values represent mean \pm SEM of three observations. Values represent mean \pm SEM of three observations. Values with different alphabet superscript in a column were significantly different $(\mathrm{P}<0.05)$. Values with different figures superscript across a row were significantly different $(\mathrm{P}<0.05)$.

\subsection{Effects of D. tripetala leaf aqueous extract on the histology of the thyroid gland}

Figure 1 showed photomicrographs of histological sections of the thyroid gland from extract treated rats and control rats. The sections of thyroid gland from normal control, standard control and extract treated groups (groups $1,3,4,5$ and 6) revealed a typical histological picture of the thyroid gland. The glands were composed of spherical follicles lined with a single layer of tall columnar epithelial cells with rounded nuclei surrounding a lumen filled with gel-like viscous iodine-rich material called colloid exhibiting serrated or vacuolated peripheral edges. However, the section of the thyroid gland from the hyperthyroid control group (group 2) showed a marked change from the normal thyroid gland. The change observed involved the absence of colloid in some follicles (empty follicles-arrows). In addition, some follicles are lined with low columnar epithelial cells. The photomicrographs of extract treated groups showed no histological difference when compared to the normal control and standard control groups. However, the photomicrograph from hyperthyroid control group showed a histological difference (absence of colloid in some follicles) when compared to other control groups and extract treatment groups. 


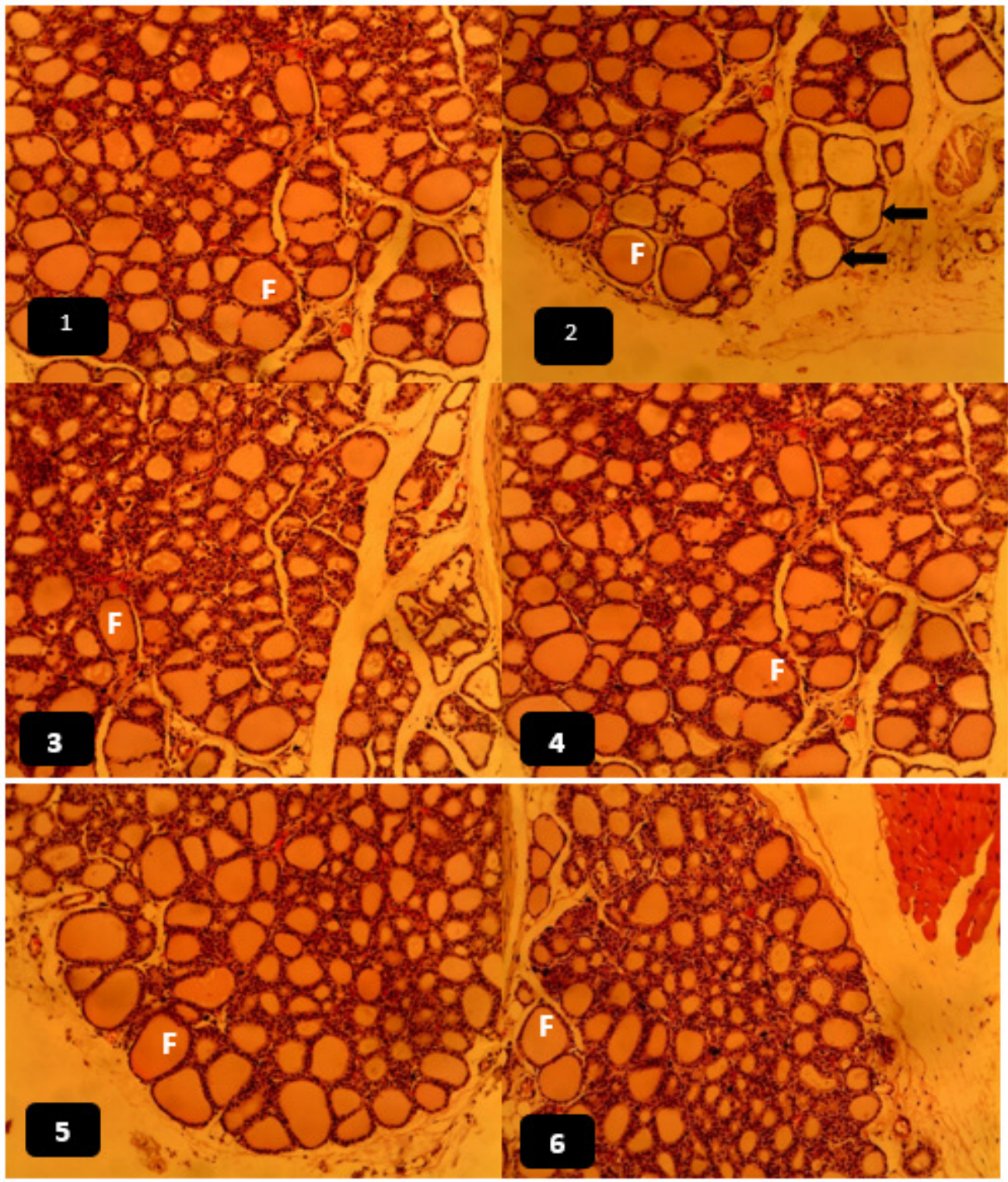

Figure 1. Photomicrograph of sections of thyroid gland from experimental groups 1 (normal control group), 2 (hyperthyroid control group), 3 (standard control group), 4 (100 $\mathrm{mg} / \mathrm{kg}$ extract dosed group), 5 (250 $\mathrm{mg} / \mathrm{kg}$ extract dosed group) and $6(500 \mathrm{mg} / \mathrm{kg}$ extract dosed group) showing follicles (F) containing colloids. See the black arrows showing absence of colloid in some follicles in group 2 (hyperthyroid group). H and E x 400 Mag.

\section{Discussion}

Based on our cage-side observations, there was no negative clinical change manifested during the acute toxicity test. Rats administered high dose of the aqueous $D$. tripetala leaf extract displayed no negative clinical change with regards to skin and fur even the eyes of the testing animals within the testing time. Additionally, there was no convulsion, lethargy, salivation, diarrhea, and sign of acute toxicity nor mortalities even at the highest dose $(5000 \mathrm{mg} / \mathrm{kg}$ ) administered. We therefore report that aqueous $D$. tripetala leaf extract could possess low oral acute toxicity in rats. This finding corroborates Anosike et al. 2016.

Interest in medicinal plants is as a result of their richness in secondary metabolites such as alkaloids, glycosides, flavonoids, steroids, tannins, and saponins (Rohini \& Padmini 2016). These phytochemical constituents are known to exhibit medicinal as well as physiological activities Ayoola et al., 2008. The phytochemical screening of the aqueous $D$. tripetala leaf extract in our present study revealed the presence of these secondary metabolites. This finding is in consonance with that of (Okoronkwo et al. 2015).

There was an observed variation in dose and duration dependent results especially in parameters such as body weight, LDL-C, TG, TSH and $\mathrm{T}_{4}$ at day 0 due to age differences among animals used. This variation caused a little inconsistency in some results. The extract $(100 \mathrm{mg} / \mathrm{kg}$ and $500 \mathrm{mg} / \mathrm{kg}$ ) proved to be most proficient (Day 7) by 
normalizing the body weight of treated rats (concentration based effect). Similarly, $500 \mathrm{mg} / \mathrm{kg}$ showed the most effective duration-based effect by normalizing the body weight of treated rats compared with normal control. These results are in line with (Bhaigyabati et al. 2012). The observed decrease in T3 and T4 levels and increase in TSH level among the $D$. tripetala leaf extract treated rats could be due to the substantial presence of flavonoids in extract (Sartelet et al. 1996). This is in agreement with the findings of (Bhaigyabati et al. 2012; Donzelli et al. 2016).

Research has proven that some plants possess antihypercholesterol, anti-obesity properties and ability to reduce body weight (Ejere \& Adegoke, 2005; Nnamonu et al. 2013; Nnamonu et al. 2018). In this study, whereas hyperthyroidism resulted in a significant decrease $(\mathrm{P}<0.05)$ in the mean total cholesterol, LDL-C and triglyceride levels, a significant increase was recorded $(\mathrm{P}<0.05)$ in $\mathrm{HDL}-\mathrm{C}$ level in the experimental rats. However, treatment with standard drug and different concentrations of aqueous $D$. tripetala leaf extract $(100 \mathrm{mg} / \mathrm{kg}, 250 \mathrm{mg} / \mathrm{kg}$ and $500 \mathrm{mg} / \mathrm{kg}$ ), significantly increased total serum cholesterol and TG levels while HDL-C significantly decreased. This observation is in agreement with (Asvold et al. 2007). There could be various physiological causes that led to the above result.

Triiodothyronine (T3) causes an up-regulation of LDL receptors, controls the sterol regulatory elementbinding protein-2, which in turn regulates LDL receptor's gene expression and protects LDL from oxidation (Rizos et al. 2011). Similarly, our present result on total cholesterol, LDL-C, triglyceride levels, and HDL-C could be attributed to the presence of some phytochemicals in the extract that have beneficial effects on blood cholesterol levels. Flavonoids have been reported to prevent the oxidation of low-density lipoprotein, low blood levels of cholesterol and triglycerides thereby reducing the risk for the development of atherosclerosis (Subramani \& Casimir 2002). Saponins bind with bile salt and cholesterol in the intestinal tract, preventing its re-absorption resulting in a reduction of blood cholesterol (Oyewole \& Akingbala 2011).

Notably, a photomicrograph of sections of the thyroid gland from experimental groups showed the absence of colloid in some follicles in hyperthyroid control. However, no remarkable histologic differences occurred in normal control, standard control and extract dosed groups. This extract effects on histology of thyroid gland may be attributed to healing properties of its phytochemical components.

\section{Conclusion and Recommendation}

Conclusively, aqueous Dennettia tripetala leaf extract seems to possess potential for ameliorating post induced hyperthyroidism effects in rat. The groups treated $250 \mathrm{mg} / \mathrm{kg}$ and $500 \mathrm{mg} / \mathrm{kg}$ Dennettia tripetala leaf aqueous extract evidently showed better result than $100 \mathrm{mg} / \mathrm{kg}$. Further researches on Dennettia tripetala leaf aqueous extract on hyperthyroidism is recommended to ensure reproducibility of our findings and new findings. Additionally, the bioactive ingredients in the leaves should be properly investigated in further experiments to explore the reproducibility of their activities in clinical trials.

\section{Competing interest's disclaimer}

Authors have declared that no competing interests exist. The products used for this research are commonly and predominantly use products in our area of research and country. There is absolutely no conflict of interest between the authors and producers of the products because we do not intend to use these products as an avenue for any litigation but for the advancement of knowledge. Also, the research was not funded by the producing company rather it was funded by the personal efforts of the authors.

\section{REFERENCES}

Anaga, A. O. \& Asuzu, I. U. (2010), Antihyperglycaemic properties of the ethyl acetate extract of Dennettia tripetala in diabetic rats. Journal of Complementary and Integrative Medicine 10:1553-1558.

Anosike, C. A., Okagu, I. U. \& Ona, K. U. (2016), Phytoconstituents, acute toxicity study and protective effect of ethanol extract of Dennettia tripetala seed against aspirin-induced ulcer in rats. International Journal of Advanced Science and Research 1(4):1-6.

Asvold, B. O., Vatten, L. J., Nilsen, T. I. \& Bjoro, T. (2007), The association between TSH within the reference range and serum lipid concentrations in a population-based study The HUNT Study. European Journal of Endocrinology 156(2):181-186.

Ayoola, G. A., Coker, H. A. B., Adesegun, S. A., Adepoju-Bello, A. A., Obaweya, K., Ezennia, E. C. \& Atangbayila, T. O. (2008), Phytochemical screening and antioxidant activities of some selected medicinal plants used for malaria therapy in south western Nigeria. Tropical Journal of Pharmaceutical Research 7(3):1019-1024.

Bhaigyabati, T., Ramya, J. \& Usha, K. (2012), Effect of methalolic extract of sweet corn silk on experimentally induced hyperthyroidism in swiss albino rat. International Research Journal of Pharmacy 3(3):241-245.

Case, O. (2005), An assessment of medicinal hemp plant extracts as natural antibiotic and immune modulation phytotherapies. Master's Thesis, South African Herbal Science and Medicine Institute, University of the Western Cape. 
Derrell, C. (1996), Guide for the care and use of laboratory animals. Institute of Laboratory Animal Resources. National Academy Press, Washington DC.

Donzelli, R., Colligiani, D., Kusmic, C., Sabatini, M., Lorenzini, L., Accorroni, A., Nannipieri, M., Saba, A., Iervasi, G. \& Zucchi, R. (2016), Effect of hypothyroidism and hyperthyroidism on tissue thyroid hormone concentrations in rat. European Thyroid Journal 5:27-34.

Ejere, V. C. \& Adegoke, J. A. (2005), Seasonal testicular histology and reproductive cycle of the Rainbow Lizard, Agama agamaagama, L, (Agamidae, Reptilia) in Ile-Ife, South Western Nigeria. Animal Research International 2(3):393-398.

Ejere, V. C., Ogbuke, E. F., Nnamonu, E. I., Ikele, B. C. \& Nweze, B. C. (2018), Evaluation of Anti-Obesity Potentials of Sphenostylis stenocarpa Ethanolic Seed Extract. Annual Research \& Review in Biology 26(3):19.

Golden, S. H., Robinson, K. A., Saldanha, I., Anton, B. L. \& Adenson, P. W. (2009), Clinical review: Prevalence and incidence of endocrine and metabolic disorders in the United States: a comprehensive review. The Journal of Clinical Endocrinology \& Metabolism 94:1853-1878.

Ihemeje, A., Ojinnaka, M. C., Obi, K. C. \& Ekwe, C. C. (2013), Biochemical evaluation of Pepper fruit (Dennettia tripetala) and its use as substitute for giner in Zobo drink production. Academic Research International 4:513521.

Ikpi, D. E. \& Nku, C. O. (2008), Effect of ethanolic extract of Dennettia tripetala fruit on haematological parameters in albino Wistar rats. Nigerian Journal of Physiological Sciences 23(1-2):13-7.

Iseghohi, S. O. (2015), A Review of the uses and medicinal properties of Dennettia tripetala (Pepper fruit). International Journal of Medical Sciences 3:104-111.

Lorke, D. (1983), A new approach to practical acute toxicity testing. Journal Archeology and Toxicology 54:275287.

Newman, D. J., Cragg, G. M. \& Snader, K. M. (2003), Natural Products as Sources of New Drugs over the Period 1981-2002.Journal of National Products 66(7):1022-1037.

Nnamonu, E. I., Ejere, V. C., Ejim, A. O., Echi, P. C., Egbuji, J. V., Eze, T. R. \& Eyo, J. E. (2013), Effects of Hibiscus Sabdariffa Calyces Aqueous Extract on Serum Cholesterol, Body Weight and Liver Biomarkers of Rattus Novergicus. Int. J Indigenous Med Plants 46(4):1405-1411.

Nnamonu, E. I., Ejere, V. C., Mbegbu, E. C., Ezechukwu, C. S. \& Ejim, A. O. (2018), Effects of methanolic calyx extract of Hibiscus sabdariffaon body weight, blood cholesterol and liver marker enzymes in Wistar rats. Journal of Medicinal Plants Research 12(26):427-434.

Nwankpa, P. 1., Ekweogu, C. N., Egwurugwu, J. N., Chukwuemeka, O. G., Etteh, C. C., Ugwuezumba, P. C. \& Emengagha, F. C. (2018), Assessment of Kidney Function Indices in Male Albino Wistar Rats Administered Ethanol Stem Extract of Dennettia tripetala (Pepper fruit). Biochemistry \& Pharmacology: Open Access Biochemistry \& Pharmacology: Open Access 7(1):1-4.

Okoronkwo, N. E., Mbachu, K. A. \& Nnaukwu, N. C. (2015), Evaluation of chemical compositions and in vitro antimicrobial activity of extracts from Dennettia tripetala leaves. International Journal of Plant Science and Ecology 1(3):72-80.

Oyemitan, I. A., Iwalewa, E. O., Akanmu, M. A., Asa, S. O. \& Olugbade, T. A. (2006), The abusive potentialof habitual consumption of the fruits of Dennettia tripetala G. Baker (Annonaceae) among the people in Ondo Township (Nigeria). Nigerian Journal of Natural Products Medicine 10:55-62.

Oyemitan, I. A., Iwalewa, E. O., Akanmu, M. A. \& Olugbade, T. A. (2008), Antinociceptive and anti-infammatory effects of essential oil Dennettiatripetala G. Baker (Annonaceae) in rodents. African Journal Traditional, Complementary and Alternative Medicine 5:353-365.

Oyewole, O. I. \& Akingbala, P. F. (2011), Phytochemical analysis and hypolipidemic properties of Jatro phatanjorensis leaf extract. European Journal of Medicinal Plants 1(4):180-185.

Peter, K. J., Amsler, C. D., Amsler, M. O., McClintock, J. B., Dunber, R. B. \& Baker, B. J. A. (2005), Comparative analysis of the nutritional and elemental composition of macroalgae from the Western Antarctic Peninsula. Phycologia 44:453-463.

Rizos, C. V., Elisaf, M. S. \& Liberopoulos, E. N. (2011), Effects of thyroid dysfunction on lipid profile. Open Cardiovascular Medicine Journal 5:76-84.

Rohini, M. V. \& Padmini, E. (2016), Preliminary phytochemical screening of selected medicinal plants of polyherbal formulation. Journal of Pharmacognosy and Phytochemistry 5(5):277-282.

Sartelet, H., Serghat, S., Lobstein, A., Ingenbleek, Y., Anton, R., Petitfrère, E., Aguie-Aguie, G., Martiny, L. \& Haye, B. (1996), Flavonoids extracted from fonio millet (Digitariaexilis) reveal potent antithyroid properties. Nutrition 12(2):100-106.

Sood, R. (2006), Medical Laboratory Technology. Jaypee Brothers medical Publishers Limited. New Delhi, P 675.

Subramani, S. \& Casimir, C. A. (2002), Flavonoids and antioxidant activity of Georgia grown Vidalia onions. Journal of Agricultural and food chemistry 50(19):5338-5342. 